\title{
SPONTANEOUS COMMON BILE DUCT PERFORATION ON THE BACKGROUND OF PANCRAETIC LITHIASIS : A CASE REPORT AND REVIEW OF THE LITERATURES
}

\author{
FAROOQ MAA ${ }^{1}$, RAHMAN MAM $^{2}, \mathrm{KABIR}^{3}{ }^{3}$, BHUIYAN MAH ${ }^{4}$, SAJID MM ${ }^{5}$, TALUKDER R ${ }^{6}$, \\ CHOWDHURY TK ${ }^{7}$, BANU T ${ }^{8}$
}

\begin{abstract}
Spontaneous bile duct perforation (SBDP) is a relatively rare condition. The cause of SBDP is still speculative. Presentation, site of the perforation and management varies. Pancreatic lithiasis ( $P L$ ) is a very rare entity in childhood. Biliary perforation on the background of PL is still a rare event. This is a case report of 10 years old girl presented as acute abdomen with theclinical diagnosis of acute on chronic pancreatitis, for which conservative treatment followed by laparotomy was carried out.Common bile duct ( CBD ) perforation was found near it's junction with cystic duct. $T$ tube was
\end{abstract}

1. Dr. Md. Abdullah AI Farooq, FCPS, MS, Assistant professor, Department of Pediatric Surgery, Chittagong Medical College\& Hospital, Chittagong, Bangladesh.

2. Dr. M A Mushfiqur Rahman, FCPS, MS, Assistant professor, Department of Pediatric Surgery, Chittagong Medical College\& Hospital, Chittagong, Bangladesh.

3. Dr. Mahfuzul Kabir, MS, Registrar, Department of Pediatric Surgery, Chittagong Medical College \& Hospital, Chittagong, Bangladesh.

4. Dr. Md. Akbar Husain Bhuiyan, MS, Assistant professor, Department of Pediatric Surgery, Chittagong Medical College\& Hospital, Chittagong, Bangladesh.

5. Dr. Md. Minhajuddin Sajid, Assistant professor, Department of Pediatric Surgery, Chittagong Medical College\& Hospital, Chittagong, Bangladesh.

6. Dr. Rupam Talukder, MBBS, MS final part student ( Pediatric Surgery), Chittagong Medical College \& Hospital, under ChittagongUniversity, Chittagong, Bangladesh.

7. Dr. Tanvir Kabir Chowdhury, MBBS, MS final part student ( Pediatric Surgery ), Chittagong Medical College \& Hospital, under Chittagong University, Chittagong, Bangladesh

8. Professor Tahmina Banu, MS, FRCS, Head, Department of Pediatric Surgery, Chittagong Medical College\& Hospital, Chittagong, Bangladesh.

Correspondence to : Dr. Md. Abdullah AI Farooq, FCPS, MS, Assistant professor, Department of Pediatric Surgery, Ward 11-B, Chittagong Medical College \& Hospital, Chittagong 4000, Bangladesh. Mobile: $+88-01815002188$, E-mail: farooq71bd @yahoo.com instituted and a drain was kept in situ. Postoperative period ( POP )was uneventful. T- Tube Cholangiogram was carried out on $12^{\text {th }}$ postoperative day( POD ) that showed dye had passed to duodenum easily and patency and caliber of the CBD was found normal. Multiple stones were noted in the vicinity. No apparent cause of CBD perforation was found. After one month, in follow up visit the patient was found alright. She was asked to come after 3 months for further evaluation. The plan is to evaluate the patient's conditions clinically and biochemically and to do an MRCP to see details of pancreatic pathology. As SBDP in children with $P L$ is a rare condition, high index of suspicion is needed for early diagnosis and proper management of such patient.

Key words: Spontaneous bile duct perforation, Pancreatic lithiasis

\section{Introduction}

Spontaneous bile duct perforation (SBDP) is a relatively rare condition with about 150 cases as of 2003. Although many theories have been proposed, the cause of bile duct perforation is speculative; distal bile duct obstruction, pancreatic fluid reflux up the common bile duct, congenital weakness of the common bile duct, a localized malformation of the wall of the common duct and pancreatic biliary duct. Biliary ascites from spontaneous bile duct perforation typically occurs in infants and toddlers from birth up to about 2 years of age, but it can occur in older children as well ${ }^{1}$.In a series of 11 patients $^{2}$ the presentation was generalized/ localized biliary peritonitis and secondary biliary stenosis. In a major review of 54 reported cases ${ }^{3}$ the clinical presentation was peritonitis with or without ascites. Site of the perforation and management varies. Jadhav V, Gupta 
$\mathrm{R}$, Parelkar et al. reported a case of 10 years old child with spontaneous cystic duct perforation that was confirmed by Endoscopic Retrograde Cholangiopancreatography ( ERCP ) and biliary stent was introduced ${ }^{4}$.According toThomas, ERCP and biliary stenting aresuccessful in the management of these patients ${ }^{5}$. Three infants with idiopathic perforation of the extra hepatic bile duct were evaluated by Banaris et al. These patients presented with jaundice, biliary ascites and peritonitis. After laparotomy, they found perforations in the bile duct. They repaired the perforations and simple abdominal drainage instituted. They recommended that perforation of the bile duct should be suspected in every neonate with jaundice and ascites and simple drainage is the treatment of choice provided the distal obstruction is excluded ${ }^{6}$. Thomas C. Moore reported an infant with spontaneous common hepatic duct ( CHD) perforation at its junction with cystic duct. He managed the case successfully with Roux-en-y hepaticojejunal anastomosis with remnant of $\mathrm{CHD}{ }^{7}$.Pancreatic lithiasis ( $P L$ ) is a very rare entity in childhood and most of the PL series in English language literature contain many segmented and partial description that are sometime difficult to correlate ${ }^{8}$. Although pancreatitis is uncommon in childhood it must be considered in every child with unexplained acute or recurrent abdominal pain. PL are one of the most frequent causes of chronic pancreatitis ${ }^{9}$. PL has two forms; calcilithiasis, generally due to nutritional disorder and proteic lithiasis, the heriditary cases of which shows autosomal dominant transmission ${ }^{10}$. ERCP is being used with increasing frequency in the pediatric population for both diagnostic and therapeutic purpose ${ }^{11}$. Since the complication of ERCP is higher in childhood even in experienced hand, MRCP should be the priority for diagnostic purpose ${ }^{12}$. After extensive literature search, no case of biliary perforation in pediatric patients with pancratic lithiasis was noted. So our case is unique in that sense.

\section{Case report}

A 10 years old girl from Moheshkhali, a remote island off the shore, was admitted in the department of Pediatric Surgery, Chittagong Medical College and Hospital, Chittagong, Bangladesh on $12^{\text {th }}$ January 2012; with the problems of severe upper abdominal pain associated with nausea and vomiting for three days and fever for two days. The pain was, diffuse, constant, severe, having no aggravating or relieving factors or radiation.Pain was associated with nausea and patient used to vomit 3- 4 times a day. Vomitus was, bile stained and effortless. The child had also been suffering from continuous fever with chills and rigor for the last couple of days. She had no desire to take food, her bowel did not move and she passed small amount of high coloured urine. The girl had previous history of upper abdominal pain and weight loss for the last one and a half month. The pain was dull aching in the upper abdomen persisting for few hours, occasionally radiating to the back and the child found it comfortable on leaning forwards or taking drugs. The pain was not associated with vomiting. The patient had complained that she gradually lost her weight for the last one and a half month. She did not give any history of loose frothy stool. For these complaints she did not consulted with any qualified doctors. There was no history of trauma, instrumentation or jaundice. The girl was immunized, took anthelmintic regularly but did not take any antiulcer drug. On examination the patient was conscious and cooperative having an average built. She was anxious, ill looking and dehydrated. Her temperature was $100^{\circ} \mathrm{F}$, pulse rate was 106 beats/ minute, respiratory rate was $22 /$ minute and systolic/ diastolic blood pressure recorded $90 / 60 \mathrm{~mm}(\mathrm{Hg})$. But the patient was not icteric, not anaemic with no signs of malnutrition. No accessible lymph node was palpable. Abdominal examination revealed that abdomen was distended with no engorged vein or visible peristalsis. Umbilicus was normal and both the groins were normal. Generalized abdominal muscle guard and rigidity were noted. It was diffusely tender with rebound tenderness. By gentle percussion ascites was elicited but upper border of liver dullness was not obliterated Bowel sound was sluggish. It was not possible to palpate solid abdominal organs. Digital rectal examination revealed no abnormality. Other systems appeared normal clinically. Baseline laboratory and imaging studies were carried out. Complete blood count (CBC) showed leukocytosis (11800/ cubic mm ). Serum amylase was $1204 \mathrm{U} / \mathrm{L}$ ( Normal 56-190 U/L ) and urinary amylase was 360 U/ L ( Normal 23-85 U/ L ). Pancreatic lipase was also found elevated ( $204 \mathrm{U} / \mathrm{L}$, normal value 5- 60 ). Serum electrolytes, serum calcium, random blood sugar and urinalysis were normal. Plain abdominal radiograph in erect posture anteroposterior view ( $X$ ray $a b . A / P$ ) showed gas distended loops of intestine and some haziness at the lower abdomen. Abdominal Ultrasound( US ) showed gas distended loops of intestine and significant amount of free fluid in the abdomen. X-ray or US did not give any information about the pancreatic pathology. 


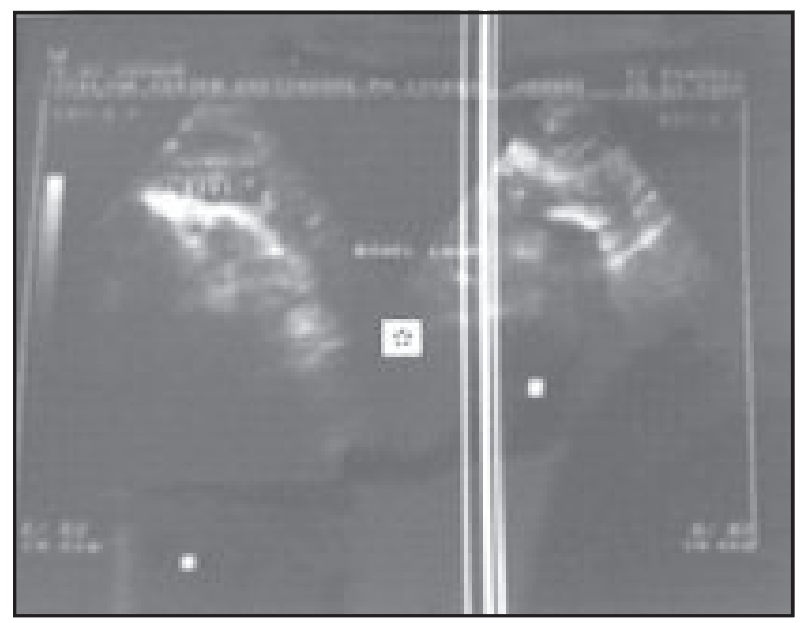

Fig-1: USG showing gas distended bowel loop(asterix).

Considering the history, clinical findings and investigations the patient was diagnosed as a case of acute on chronic pancreatitis The patient underwent conservative treatment with complete bed rest, intravenous ( IV ) fluid, nasogastric ( NG ) suction, parenteral antibiotics, $\mathrm{H}_{2}$-blocker, analgesic, urethral catheter and oxygen inhalation. All the vital signs with urine output and abdominal conditions including abdominal girth were monitored six hourly to ensure the adequacy of the conservative treatment and to observe the improvement of the symptoms and signs. After two days observation it was found that symptoms had worsen. She showed no desire to take food, her bowel did not move. Abdominal pain remained severe associated with nausea, fever rose up to $103^{\circ} \mathrm{F}$, but there was no vomiting after NG suction. Urine colour and volume became normal and dehydration was corrected and BP came towards normal. Her pulse rate increased to 126 beats /minute, respiratory rate was 26 / minute. Abdominal distension (girth measured $5 \mathrm{~cm}$ more than before), muscle guard and rigidity increased while tenderness and rebound tenderness worsened. No bowel sound was found and the abdomen became silent. Decision for laparotomy was taken. After abdominal exploration bilious ascites was noted and fibrinous exudate found on the gut. Fluid was taken and sent for bacteriological culture and sensitivity ( CS). After proper cleansing, a gangrenous perforation with margin measuring about $0.5 \mathrm{~cm}$ diameter was found in the common bile duct near it's junction with cystic duct. Duodenum, gall bladder (GB) and liver were healthy. Patency of CBD was assessed by $10 \mathrm{fr}$ feeding tube which was introduced up to the duodenum. Normal saline also passed freely up to duodenum. No stone was found in GB or CBD. Margin of the perforation was trimmed and T- tube was inserted into the common bile duct. Peroperative cholangiogram was not possible as $\mathrm{C}$ - Arm was not available. Peritoneal toileting was done and a drain kept in situ.

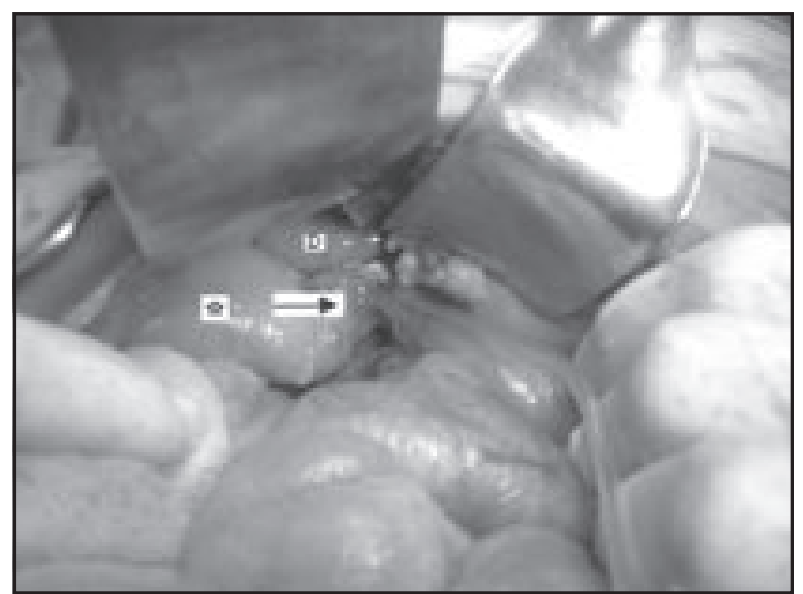

Fig-2: Per operative view(arrow= feeding tube in perforated $C B D$, Asterix= gall bladder, Arrowhead= liver).

Her post-operative recovery was uneventful. T- Tube Cholangiogram was carried out on $12^{\text {th }}$ post-operative day (POD) that showed dye had passed to duodenum easily and patency and caliber of the CBD was found normal. Multiple stones were noted in the vicinity of the pancreas. The T- tube was removed on $14^{\text {th }}$ POD.

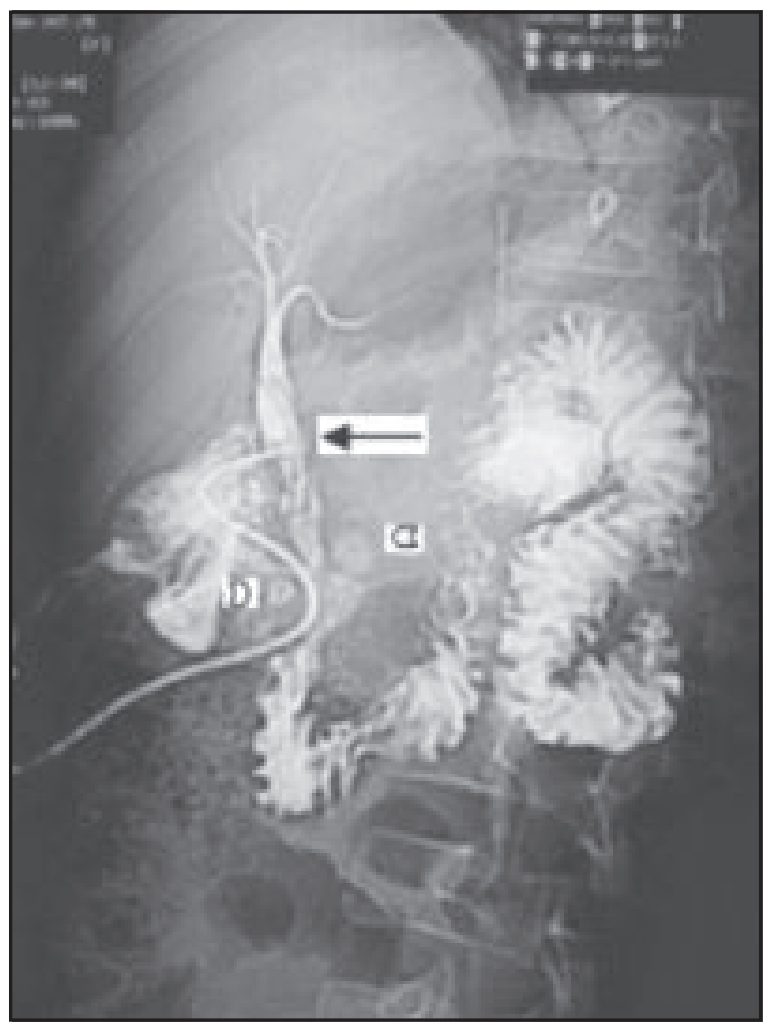

Fig-3: $T$ tube cholangiogram ( arrow heads= pancreatic stones, arrow $=T$ tube in $C B D$ ) 
On $15^{\text {th }} P O D, X$-ray ab. A/P was done to confirm the pancreatic calculi.

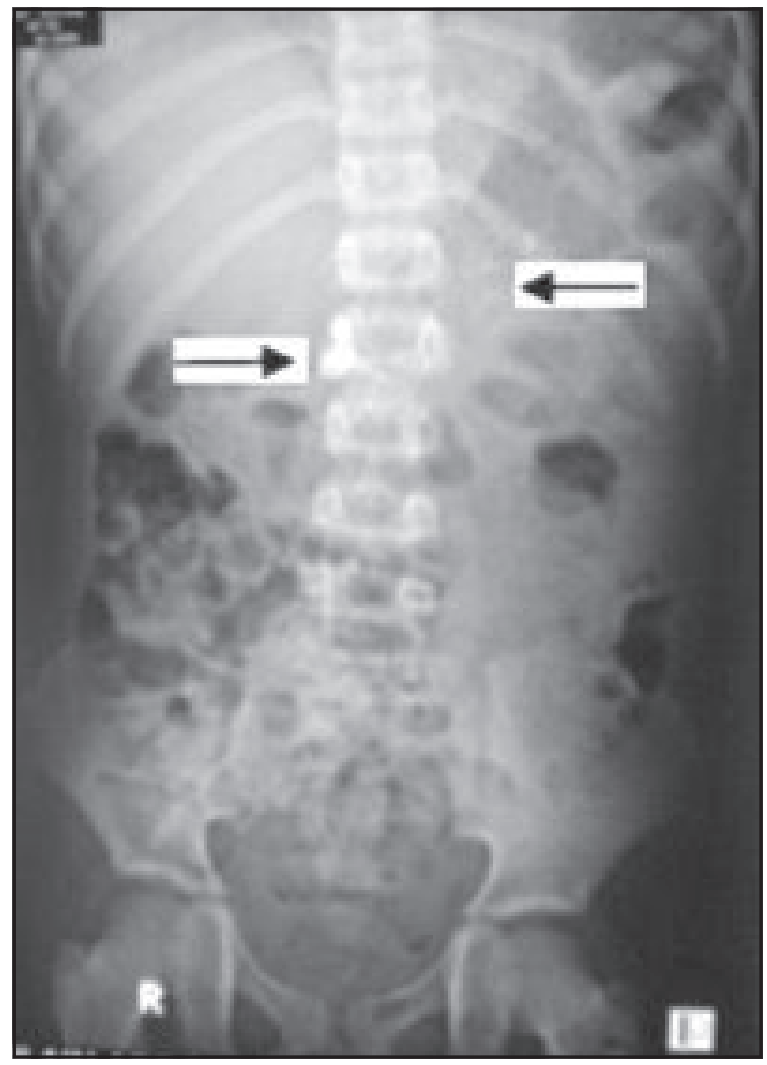

Fig-4: Plane Xray abdomen (arrow = pancreatic stones)

No growth of organism was noted in CS report. The patient was discharged on $16^{\text {th }}$ POD and followed up after one month. Patient was alright and paracetamol was prescribed for pain control. The patient was asked to come after 3 months for further evaluation. The plan is to evaluate the patient's symptoms clinically, biochemically and to do an MRCP to see details of pancreatic pathology.

\section{Discussion}

Spontaneous bile duct perforation (SBDP) is a relatively rare condition. The cause of bile duct perforation is speculative as are distal bile duct obstruction, pancreatic fluid reflux up the common bile duct, congenital weakness of the pancreatic biliary duct system. Biliary ascites from spontaneous bile duct perforation typically occurs in infants and toddlers from birth upto about 2 years of age, but it can occur in older children as well ${ }^{1}$. In patients, the presentation was generalized/ localized biliary peritonitis ${ }^{2}$. In 54 reported casesthe clinical presentation was peritonitis with or without ascites ${ }^{3}$. Site of the perforation and management varies. Acase of 10 years old child with spontaneous cystic duct perforation was confirmed by ERCP and biliary stent was introduced ${ }^{4}$.ERCP and biliary stenting are successful in the management of these patients ${ }^{5}$.Three infants with idiopathic perforation of the extra hepatic bile duct was evaluated which presented with jaundice, biliary ascites and peritonitis. After laparotomy, they found perforations in the bile duct which were repaired with institution of simple abdominal drainage. They recommended that perforation of the bile duct should be suspected in every neonate with jaundice and ascites and simple drainage is the treatment of choice provided the distal obstruction is excluded ${ }^{6}$. Thomas $\mathrm{C}$. Moore reported an infant with spontaneous common hepatic duct perforation at its junction with cystic duct. He managed the case successfully with Roux-en-y hepaticojejunal anastomosis with remnant of $\mathrm{CHD}^{7}$. PL is a very rare entity in childhood ${ }^{8}$. Although pancreatitis is uncommon in childhood it must be considered in every child with unexplained acute or recurrent abdominal pain. $P L$ is one of the most frequent causes of chronic pancreatitis ${ }^{9}$. PL has two forms- calcific and proteic ${ }^{10}$. ERCP is being used with increasing frequency for both diagnostic and therapeutic purpose ${ }^{11}$ but MRCP is preferable to ERCP for diagnostic purpose ${ }^{12}$. After extensive literature search, no case of biliary perforation in pediatric patients with $\mathrm{PL}$ was noted.

\section{Conclusion}

Although spontaneous biliary perforation in children with pancreatic lithiasis is a rare condition, high index of suspicion is needed for early diagnosis and proper management of such patient.

\section{References}

1. Eugene DM III. Ascites. In Grosefeld JL, O' neill Jr, Fonkalsrud EW, Coran AG (eds), Pediatric Surgery, Philadelphia, 2006; p. 1409

2. Chardot $\mathrm{C}$, Iskandarani $\mathrm{F}$ et al. Spontaneous perforation of the biliary tract in infancy: a series of 11 cases. Eur J Pediatr Surg 1996; Dec: 6(6):341-6.

3. Lilly JR, Weintraub WH et al. Spontaneous perforation of the extrahepatic bile ducts and bile peritonitis in infancy. Surgery, 1992; 111:237-9.

4. Jadhav V, Gupta R, Parelkar SV, et al. Endoscopic management of spontaneous 
perforation of a cystic duct. Eur J Pediatr Surg 2009;19(3): 194-6 .

5. Thomas A. J Pedsurg. 2009.09.049

6. Banaris S A, Bahader A, Nazakatgoo N et al. Idiopathicperforation of the extra hepatic bile duct, Pathogenesis, diagnosis and management. J Ped Surg, 1993; 28(7):

7. Thomas C. Moore. Massive bile peritonitis in infancy due to spontaneous bile duct berforation with portal vein ocllusion. Ped Surg, 1975; 10(4): 537-538

8. Yalcin S., Arbaj O., Ciftci et al. Management of pancreatic lithiasis in a 5-year-old girl. Turk J Pediatr 2008; 50: 189 -192.
9. O'Neill. Intussusception. In O'Neill JA, Rowe, Grosfeld, Fonkalsrud EW and Coran AG(eds), Pediatric Surgery , Missouri, 1998; 1185-1195.

10. Sarles H, Camarena J, Bernard JP. Hereditary protein lithiasis and calcium lithiasis: two different forms of hereditary pancreatitis. In Bull Acad Natl Med 1993; 177: 565-571.

11. Pascale P, Laberge JM. Endoscopic retrograde cholangiopancreatography in children: a surgeon's perspective. J Pediatr Surg 2001; 36 : 733-735.

12. Guelrud M. Endoscopic retrograde cholangiopancreatography in children. Gastroenterologist 1996; 4: 81-97. 